\title{
Combined prognostic nutritional index ratio and serum amylase level during the early postoperative period predicts pancreatic fistula following pancreaticoduodenectomy
}

Teruhisa Sakamoto, Takuki Yagyu, Ei Uchinaka, Masaki Morimoto, Takehiko Hanaki, Joji Watanabe, Manabu Yamamoto, Tomoyuki Matsunaga, Naruo Tokuyasu, Soichiro Honjo and Yoshiyuki Fujiwara

\begin{abstract}
Background: The aim of this study was to investigate the usefulness of the range of change in prognostic nutritional index (PNI) during the early postoperative period as a predictor of postoperative pancreatic fistula (POPF) after pancreaticoduodenectomy.

Methods: Data were retrospectively analyzed for 192 patients who underwent pancreaticoduodenectomy. Univariate and multivariate logistic regression analyses were used to evaluate perioperative variables. PNIP-Pre ${ }^{\text {ratio }}$ represented the range of change in PNI from before surgery to postoperative day (POD) 3, PNI ${ }^{\mathrm{P}-\mathrm{Pre}}$ ratio represented the range of change in $\mathrm{PNI}$ from before surgery to $\mathrm{POD} 1$, and $\mathrm{PNI}{ }^{\mathrm{P3}-\mathrm{P} 1}$ ratio represented the range of change in PNI from POD 1 to POD 3.

Results: The area under the curve (AUC) for PNI ${ }^{\mathrm{P3}-\mathrm{P} 1}$ for prediction of POPF following pancreaticoduodenectomy was $0.683(P<0.001)$, which was highest among PNI ratios and higher than PNI on POD 3. The AUC for serum amylase level on POD 1 was $0.704(P<0.001)$, which was superior to the corresponding AUC on POD 3. The AUC for the combination of $\mathrm{PNI}{ }^{\mathrm{P}-\mathrm{P} 1}$ ratio and serum amylase level on POD 1 for prediction of POPF was higher than the AUC of either indicator alone $(0.743, P<0.001)$. The combination of $P N P^{P 3-P 1}$ ratio and serum amylase level on POD 1 was an independent predictor of POPF following pancreaticoduodenectomy $(P=0.018)$.
\end{abstract}

Conclusions: The combination of the range of change in PNI from POD 1 to POD 3 and serum amylase levels on POD 1 may be useful for prediction of POPF following pancreaticoduodenectomy.

Keywords: PNI, Serum amylase level, Pancreatic fistula

\footnotetext{
* Correspondence: tesakamo@tottori-u.ac.jp

Division of Surgical Oncology, Department of Surgery, School of Medicine, Tottori University Faculty of Medicine, 36-1 Nishi-cho, Yonago 683-8504, Japan
}

C C The Author(s). 2020 Open Access This article is licensed under a Creative Commons Attribution 4.0 International License, which permits use, sharing, adaptation, distribution and reproduction in any medium or format, as long as you give appropriate credit to the original author(s) and the source, provide a link to the Creative Commons licence, and indicate if changes were made. The images or other third party material in this article are included in the article's Creative Commons licence, unless indicated otherwise in a credit line to the material. If material is not included in the article's Creative Commons licence and your intended use is not permitted by statutory regulation or exceeds the permitted use, you will need to obtain permission directly from the copyright holder. To view a copy of this licence, visit http://creativecommons.org/licenses/by/4.0/ The Creative Commons Public Domain Dedication waiver (http://creativecommons.org/publicdomain/zero/1.0/) applies to the data made available in this article, unless otherwise stated in a credit line to the data. 


\section{Introduction}

Mortality associated with pancreaticoduodenectomy has declined to less than $3 \%$ following recent advances in surgical procedures and perioperative managements; however, morbidity remains high [1]. Postoperative pancreatic fistula (POPF), which is the most frequent serious complication following pancreaticoduodenectomy, remains a problem; it may cause life-threatening complications such as intra-abdominal hemorrhage, intraabdominal abscess, and sepsis, all of which are associated with high mortality rates $[2,3]$. Despite extensive efforts to improve surgical outcomes following pancreaticoduodenectomy, no definitive method exists to completely prevent POPF. Therefore, it is necessary to predict POPF accurately during the early postoperative period after pancreaticoduodenectomy, to protect patients from possible severe adverse events.

Risk indicators have been suggested to predict the development of POPF. The prognostic nutritional index (PNI), which is calculated from routine blood tests, is a well-known nutritional indicator, a well-recognized prognostic factor in cancer, and a predictive risk factor for complications in highly-invasive surgeries [4-6]. Several studies have reported a relationship between PNI perioperatively and POPF following pancreaticoduodenectomy $[7,8]$. However, to the best of our knowledge, no reports have evaluated the usefulness of the range of change in PNI during the early postoperative period for prediction of POPF following pancreaticoduodenectomy, despite hemodynamic changes in perioperative systemic responses (e.g., inflammation and immunocompetence).

Amylase levels are also a reliable method for prediction of POPF. Drain amylase level has been generally accepted as the main diagnostic indicator of POPF in the International Study Group of Pancreatic Fistula (ISGPF) criteria, and has been used for prediction of POPF worldwide. However, some reports demonstrated that the concentration of drain amylase does not always match the severity of POPF following pancreaticoduodenectomy [9-11]. Several previous studies indicated that an elevated serum amylase level during the early postoperative period was associated with the development of POPF [12-14]. This may reflect the local response from remnant pancreatic tissue, considering that an elevated serum amylase level represents pancreatitis in the remnant pancreatic tissue or local ischemia, which leads to POPF $[15,16]$. However, the relationship between PNI (an indicator of the systemic response) and serum amylase level (an indicator of the local response), regarding prediction of POPF after pancreaticoduodenectomy, remains unclear. We speculated that the combination of the range of change in PNI and the serum amylase level, which indicate different patient responses during the early postoperative period, might be superior to using the range of change in PNI alone for prediction of POPF after pancreaticoduodenectomy.

The aim of the present study was to investigate the usefulness of the range of change in PNI during the early postoperative period for prediction of POPF following pancreaticoduodenectomy. An additional aim was to assess the predictive significance of the combination of the range of change in PNI and serum amylase level during the early postoperative period for POPF following pancreaticoduodenectomy.

\section{Patients and methods Patients}

Data for 192 consecutive patients who underwent pancreaticoduodenectomy for malignant or benign disease of the pancreatic head and periampullary region at Tottori University Hospital (Yonago, Japan) between January 2008 and July 2019 were retrospectively analyzed.

\section{Surgical procedures}

Standard pancreaticoduodenectomy or pyloruspreserving pancreaticoduodenectomy was performed for the patients enrolled in this study. Reconstruction of the digestive tract was performed using a modified Child's method in standard pancreaticoduodenectomy or the Traverso method in pylorus-preserving pancreaticoduodenectomy, with a Braun anastomosis. Pancreatojejunostomy was performed with a duct-to-mucosa anastomosis in end-to-side fashion using eight 5-0 absorbable interrupted sutures with either internal or external stents for drainage of pancreatic juice in the main pancreatic duct; for seromuscular-parenchymal anastomosis, either the modified Kakita method or the modified Blumgart method was performed using 3-0 or 4-0 nonabsorbable sutures [17]. After reconstruction of the digestive tract, closed peritoneal drainage tubes were routinely inserted at the superior and inferior sides of the pancreaticojejunostomy and behind the hepaticojejunostomy.

\section{Postoperative management}

Prophylactic antibiotics were routinely administered for 3 days, including the day of surgery, in all patients. Oral intake was usually started on postoperative day (POD) 4 unless postoperative serious complications occurred, such as paralytic ileus or severe pneumonia. No patients received preoperative nutritional support. However, nutritional support (e.g., enteral nutrition via enteral feeding tube or total parenteral nutrition) was administered to patients with poor oral intake after surgery during the perioperative period. Proton pump inhibitors were provided to patients throughout the entire postoperative course. 
The amylase concentrations in drain fluids were examined on POD 1 and 3. Concurrent bacterial culture and bacterial smear tests of the drain fluids were performed to detect infection. If the drain amylase levels on POD 3 were less than three-fold greater than serum values or less than < $1000 \mathrm{IU} / \mathrm{l}$ and bacterial smear tests of drainage fluid on POD 3 were negative, the drains were removed on POD 3 or 4 . In patients with suspected POPF with leakage of the pancreaticojejunostomy or infection, the drains were replaced on POD 7, then once per week. The drains were maintained until POPF was resolved.

POPF was defined in accordance with ISGPF criteria [18]. POPF classified as grade $\mathrm{B}$ or $\mathrm{C}$ according to ISGP $\mathrm{F}$ criteria was considered POPF in this study; patients were divided into a POPF group and a non-POPF group. The upper limit of normal serum amylase in our institution was $132 \mathrm{IU} / \mathrm{l}$.

\section{Clinicopathological variables}

Patients' medical records were retrospectively reviewed for the following clinicopathological variables: age, sex, body mass index, histological diagnosis, preoperative biliary drainage, pancreatic texture of the remnant pancreas, diameter of the main pancreatic duct, operation time, intraoperative blood loss volume, and the method of pancreaticojejunostomy. Serum amylase and Creactive protein (CRP) levels were measured on POD 1 and 3. Serum albumin concentration and absolute total lymphocyte count (as components of PNI) were also recorded before surgery and on POD 1 and 3. PNI was calculated as follows: $10 \times$ serum albumin concentration $+0.005 \times$ total lymphocyte count [4].

In the present study, the range of change in PNI during the early postoperative period was defined as the PNI ratio and the values were calculated as follows: $\mathrm{PNI}^{\mathrm{P} 3-\mathrm{Pre}}$ ratio, PNI on POD 3 divided by preoperative PNI multiplied by 100; PNI $^{\text {P1-Pre }}$ ratio, PNI on POD 1 divided by preoperative PNI multiplied by 100; and $\mathrm{PNI}^{\mathrm{P} 3-\mathrm{P} 1}$ ratio, PNI on POD 3 divided by PNI on POD 1 multiplied by 100 . The $\mathrm{PNI}^{\mathrm{P} 3-\mathrm{Pre}}$ ratio represented the range of change in PNI from before surgery to POD 3. $\mathrm{PNI}^{\mathrm{P} 1-\mathrm{Pre}}$ indicated the range of change in PNI from before surgery to POD 1 , and $\mathrm{PNI}^{\mathrm{P} 3-\mathrm{P} 1}$ indicated the range of change in PNI from POD 1 to POD 3. The cut off value of drain amylase level on POD 1 was designated as $4000 \mathrm{IU} / \mathrm{l}$, based on the information in a previous report [19].

\section{Statistical analysis}

The results of the analysis of continuous variables were expressed as median with range, and categorical variables were expressed as number (proportion, \%). The Chi-square test, Fisher's exact test, and the Mann-Whitney $U$ test were used to assess comparisons of clinicopathological variables between the two groups; correlations between two variables were evaluated using Spearman's rank correlation coefficient. Receiver operating characteristic analysis was used to evaluate the predictive significances and areas under the curves (AUCs) for prediction of POPF after pancreaticoduodenectomy. The optimal cutoff values for the PNI ratio, serum amylase level, and serum CRP level for prediction of POPF were also determined using Youden's index in receiver operating characteristic analysis.

Univariate and multivariate logistic regression analyses were performed to clarify the predictive factors for POPF after receiver operating characteristic analysis. $P<0.05$ was considered statistically significant, and all statistical analyses were performed using SPSS software (Version 24; IBM Corp., Armonk, NY, USA).

\section{Results}

POPF occurred in 54/192 (28.1\%) patients. No patients with removal of drain tubes on POD 3 or 4 required intervention such as drain re-insertion or percutaneous drainage. Postoperative enteral nutrition via enteral feeding tube was performed in 7/192 (3.6\%) patients; total parenteral nutrition was performed in 48/192 (25.0\%) patients. Nineteen of 192 (9.9\%) patients received both enteral nutrition and total parenteral nutrition during the perioperative period.

Table 1 shows the comparison of clinicopathological variables between patients with POPF (POPF group) and patients without POPF (non-POPF group). No significant correlations were observed between the POPF and non-POPF groups in terms of age, operative time, intraoperative blood loss volume, preoperative serum albumin level, preoperative total lymphocyte count, serum CRP level on POD 1, preoperative PNI, PNI on POD 1, or $\mathrm{PNI}^{\mathrm{P} 1-\mathrm{Pre}}$ ratio. The proportions of men, presence of preoperative biliary drainage, and soft pancreatic texture of the remnant pancreas were significantly higher in the POPF group than in the non-POPF group. In addition, body mass index was significantly higher in the POPF group than in the non-POPF group. The POPF group had significantly lower proportions of a histological diagnosis of pancreatic ductal adenocarcinoma and shorter diameter of the main pancreatic duct, compared with the non-POPF group. A modified Blumgart anastomosis was performed significantly more frequently in the nonPOPF group than in the POPF group. Serum and drain amylase levels on POD 1 and 3 and serum CRP levels on POD 3 were significantly higher in the POPF group than in the non-POPF group. PNI on POD $3, \mathrm{PNI}^{\mathrm{P} 3-\mathrm{Pr}}$ ratio, and $\mathrm{PNI}^{\mathrm{P} 3-\mathrm{P} 1}$ ratio were significantly lower in the POPF group than in the non-POPF group.

Receiver operating characteristic analysis revealed that the $\mathrm{AUC}$ for $\mathrm{PNI}^{\mathrm{P} 3-\mathrm{P} 1}$ ratio for prediction of $\mathrm{POPF}$ 
Table 1 Patients' clinicopathological variables

\begin{tabular}{|c|c|c|c|}
\hline Variables & POPF group $(n=54)$ & non-POPF group $(n=138)$ & $P$ value \\
\hline Age, year, median (range) & $69.7(31-84)$ & $70.9(17-86)$ & 0.604 \\
\hline Sex, male, $(n, \%)$ & $42(77.8 \%)$ & $79(57.2 \%)$ & 0.008 \\
\hline Body mass index, median (range) & $23.3(16.5-29.6)$ & $21.5(15.1-31.5)$ & 0.001 \\
\hline Histological diagnosis & & & 0.001 \\
\hline pancreatic ductal adenocarcinoma & $9(16.7 \%)$ & $64(46.4 \%)$ & \\
\hline acinar sell cancer & $0(0 \%)$ & $1(0.7 \%)$ & \\
\hline bile duct cancer & $22(40.7 \%)$ & $20(14.5 \%)$ & \\
\hline carcinoma of the papilla of Vater & $12(22.2 \%)$ & $21(15.2 \%)$ & \\
\hline IPMN & $6(11.1 \%)$ & $17(12.3 \%)$ & \\
\hline PNET & $1(1.9 \%)$ & $6(4.3 \%)$ & \\
\hline SPN & $1(1.9 \%)$ & $1(0.7 \%)$ & \\
\hline Others & $3(5.6 \%)$ & $8(5.8 \%)$ & \\
\hline Preoperative biliary drainage, present, $(n, \%)$ & $30(55.6 \%)$ & $53(38.4 \%)$ & 0.031 \\
\hline Pancreatic texture of remnant pancreas, soft, (n, \%) & $49(90.7 \%)$ & $68(49.3 \%)$ & $<0.001$ \\
\hline Diameter of main pancreatic duct, median (range), mm & $2.6(1.0-8.9)$ & $4.3(1.0-12.7)$ & $<0.001$ \\
\hline Operative time, median (range), (min) & $545(403-823)$ & $525(309-780)$ & 0.271 \\
\hline Intraoperative blood loss, median (range), (ml) & $570(64-4016)$ & $557(95-2950)$ & 0.240 \\
\hline Method of pancreaticojejunostomy, modified BA, $(n, \%)$ & $28(51.9 \%)$ & $97(70.3 \%)$ & 0.016 \\
\hline Preoperative serum albumin level, median (range), (g/dl) & $4.0(1.0-5.2)$ & $4.0(2.1-4.9)$ & 0.607 \\
\hline Preoperative total lymphocyte count, median (range), $(/ \mu l)$ & $1504(875-3168)$ & $1460(550-5382)$ & 0.338 \\
\hline Serum amylase level on POD 1, median (range), (IU/I) & $432(136-3953)$ & $246(12-2324)$ & $<0.001$ \\
\hline Serum amylase level on POD 3, median (range), (IU/I) & $102(28-665)$ & $64(8-1406)$ & 0.001 \\
\hline Drain amylase level on POD 1, median (range), (IU/I) & $8652(66-985,053)$ & $957.5(12-65,568)$ & $<0.001$ \\
\hline Drain amylase level on POD 3, median (range), (IU/I) & $1313(450-42,370)$ & $187.5(4-23,814)$ & $<0.001$ \\
\hline Serum CRP level on POD 1 (range), (mg/dl) & $7.83(4.33-17.76)$ & $7.32(1.04-20.46)$ & 0.160 \\
\hline Serum CRP level on POD 3 (range), (mg/dl) & $21.68(4.85-37.52)$ & $11.46(0.88-37.56)$ & $<0.001$ \\
\hline Preoperative PNI, median (range) & $48.4(36.6-59.8)$ & $47.7(24.6-72.9)$ & 0.439 \\
\hline PNI on POD 1, median (range) & $31.3(22.7-41.6)$ & $31.4(19.4-46.5)$ & 0.795 \\
\hline PNI on POD 3, median (range) & $29.5(23.5-40.3)$ & $32.4(22.2-49.1)$ & 0.007 \\
\hline $\mathrm{PNI}^{\mathrm{P1} \text {-Pre }}$ ratio, median (range) & $65.3(49-92)$ & $67.6(41-108)$ & 0.577 \\
\hline PNIP-Pre ratio, median (range) & $62.4(46-82)$ & $68.3(44-111)$ & 0.003 \\
\hline $\mathrm{PNI}^{\mathrm{P3}-\mathrm{P} 1}$ ratio, median (range) & $94.6(65-122)$ & $101.7(75-140)$ & $<0.001$ \\
\hline
\end{tabular}

PD pancreaticoduodenectomy, POPF postoperative pancreatic fistula, IPMN intraductal papillary mucinous neoplasm, PNET pancreatic neuroendocrine tumor, SPN solid pseudopapillary neoplasm, BA Blumgart anastomosis, $P O D$ postoperative day, CRP C-reactive protein, $P N I$ prognostic nutritional index Continuous variables are expressed as median with range

following pancreaticoduodenectomy was 0.683 $(P<0.001)$, which was highest among the PNI ratios and higher than PNI on POD 3 (Fig. 1a). Additionally, the AUC for serum amylase level on POD 1 (AUC = 0.704, $P<0.001$ ) for prediction of POPF following pancreaticoduodenectomy was higher than the corresponding AUC on POD 3 (Fig. 1b). The optimal cutoff values for $\mathrm{PNI}^{\mathrm{P} 3-\mathrm{P} 1}$ ratio and serum amylase level on POD 1 for prediction of POPF following pancreaticoduodenectomy were 100 and $268 \mathrm{IU} / \mathrm{l}$, respectively, using the highest Youden indices.
There was almost no correlation between $\mathrm{PNI}^{\mathrm{P} 3-\mathrm{P} 1}$ ratio and serum amylase level on POD $1(r=-0.184, P=$ 0.014; Fig. 2). According to these results, patients were stratified into the following three groups: $\mathrm{PNI}^{\mathrm{P} 3-\mathrm{P} 1}$ ratio $\geq 100$ and serum amylase level on POD $1<268$, score $2(n=49) ; \mathrm{PNI}^{\mathrm{P} 3-\mathrm{P} 1}$ ratio $\geq 100$ and serum amylase level on POD $1 \geq 268$ or $\mathrm{PNI}^{\mathrm{P} 3-\mathrm{P} 1}$ ratio $<100$ and serum amylase level on POD $1<268$, score $1(n=74)$; and PNI ${ }^{\mathrm{P} 3-\mathrm{P} 1}$ ratio $<100$ and serum amylase level on POD $1 \geq 268$, score $0 \quad(n=54)$. The AUC for the combination of $\mathrm{PNI}^{\mathrm{P} 3-\mathrm{P} 1}$ ratio and serum amylase level on POD 1 for 
(a)

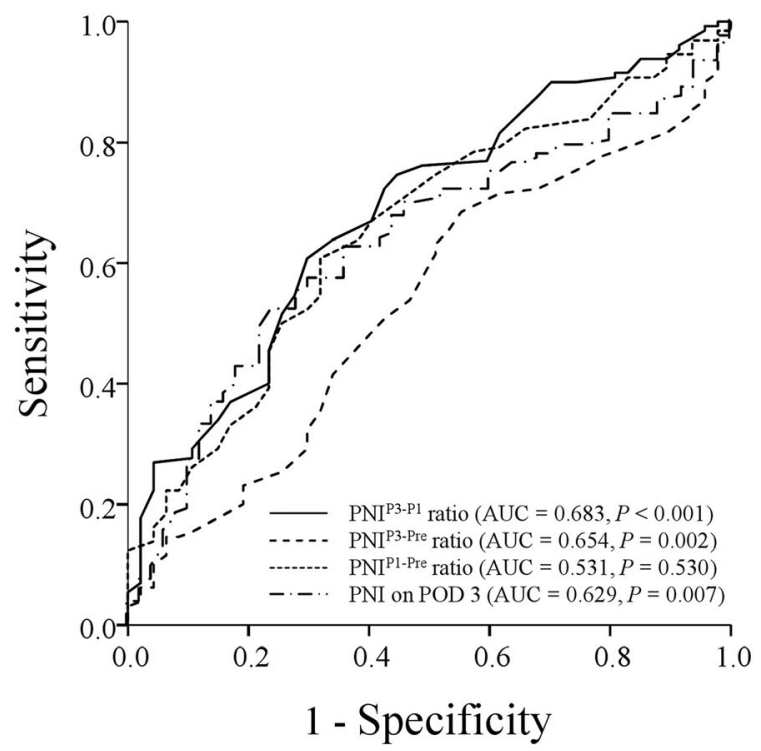

(b)

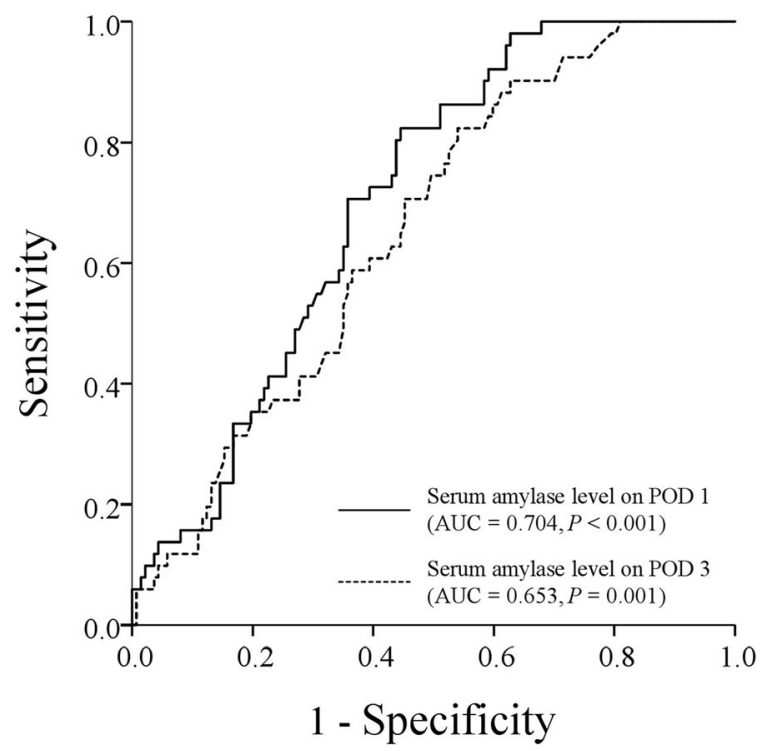

Fig. 1 Receiver operating characteristic curves for PNI on POD 3, $\mathrm{PNI}^{\mathrm{P3}-\mathrm{P1} 1}$ ratio, PNI ${ }^{\mathrm{P3}-\mathrm{Pre}}$ ratio, and PNI ${ }^{\mathrm{P}-\mathrm{Pre}}$ ratio (a), and for serum amylase levels on POD 1 and 3 (b), for prediction of clinically relevant postoperative pancreatic fistula in patients who underwent pancreaticoduodenectomy. PNI, prognostic nutritional index; AUC, area under the curve; POD, postoperative day

prediction of POPF was $0.743(P<0.001)$, indicating that the combination of $\mathrm{PNI}^{\mathrm{P3}-\mathrm{P} 1}$ ratio and serum amylase level on POD 1 was more useful than either $\mathrm{PNI}^{\mathrm{P} 3-\mathrm{P} 1}$ ratio or serum amylase level on POD 1 alone for prediction of POPF following pancreaticoduodenectomy (Fig. 3a). The
AUC for drain amylase level on POD 1 for prediction of POPF was $0.735(P<0.001)$ (Fig. 3b), which was lower than the AUC for the combination of $\mathrm{PNI}^{\mathrm{P} 3-\mathrm{P} 1}$ ratio and serum amylase level on POD 1. The AUC for serum CRP level on POD3 was $0.772(P<0.001$, Fig. 3c).

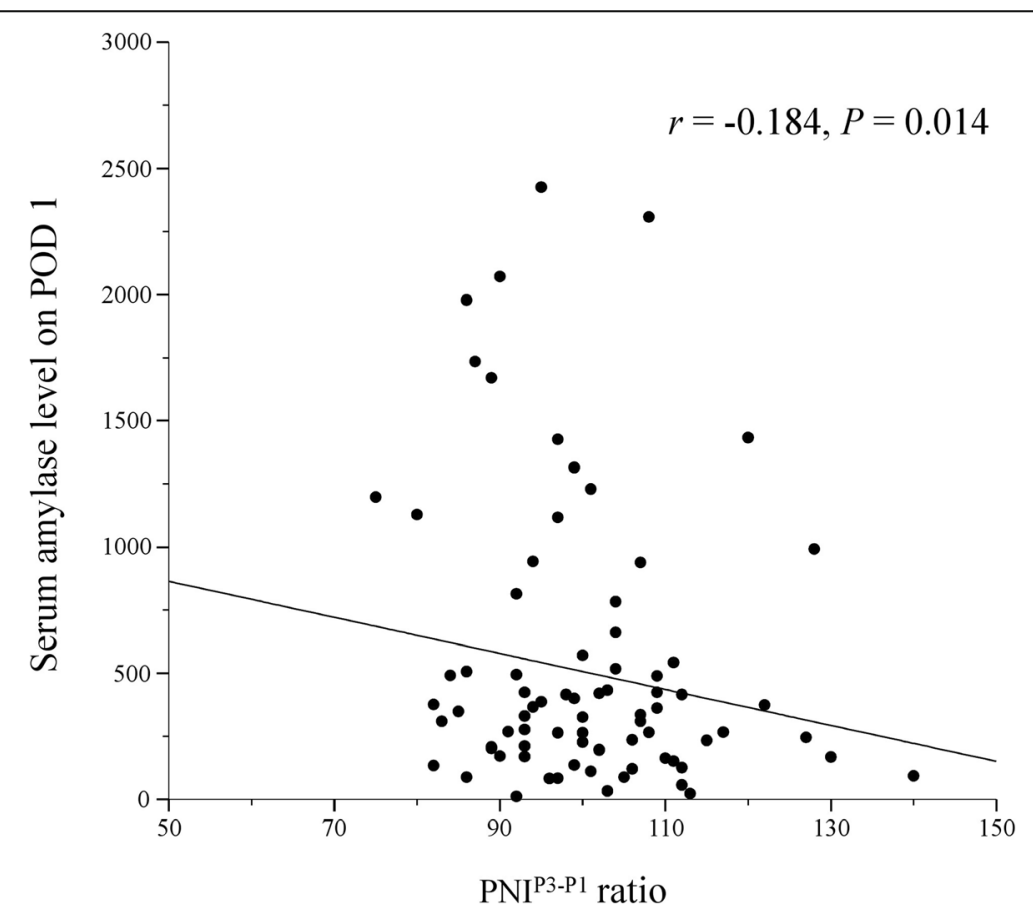

Fig. 2 Correlation between $\mathrm{PNI}^{\mathrm{P3}-\mathrm{P1}}$ ratio and serum amylase levels on POD 1. PNI, prognostic nutritional index; POD, postoperative day 


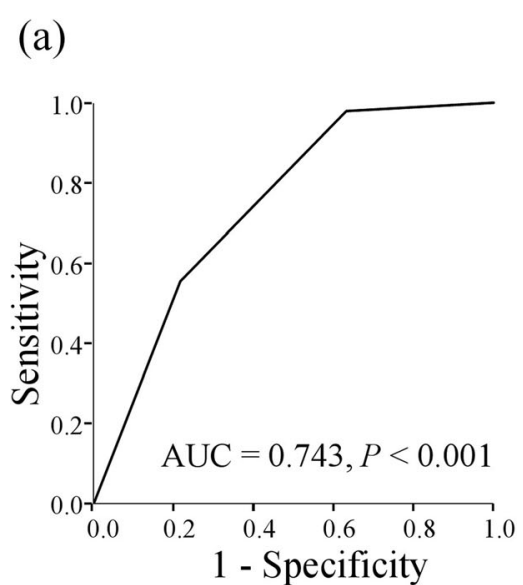

(b)

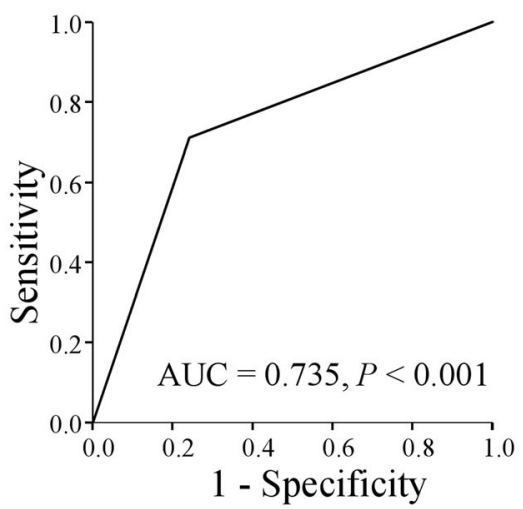

(c)

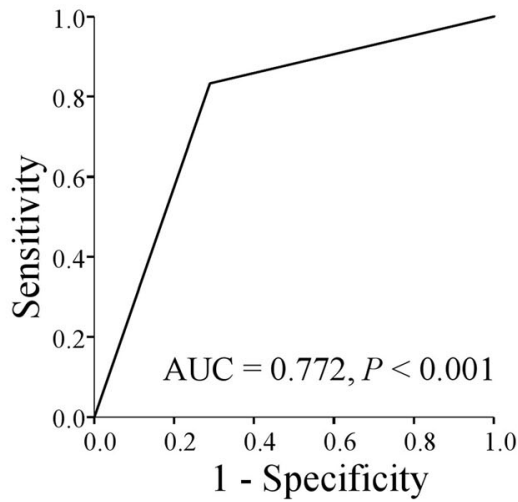

Fig. 3 Receiver operating characteristic curves for the combination of PNI ${ }^{\mathrm{P}-\mathrm{P} 1}$ ratio and serum amylase level on POD 1 (a), drain amylase level on POD 1 (b), and serum CRP level on POD 3 (c) for prediction of clinically relevant postoperative pancreatic fistula in patients who underwent pancreaticoduodenectomy. PNI, prognostic nutritional index; POD, postoperative day; CRP, C-reactive protein; AUC, area under the Curve

Finally, multivariate analysis revealed that the combination of $\mathrm{PNI}^{\mathrm{P} 3-\mathrm{P} 1}$ ratio and serum amylase level on POD 1 was an independent predictor of POPF after pancreaticoduodenectomy $(P=0.038)$, as were the presence of preoperative biliary drainage $(P=0.019)$ and serum CRP level on POD3 $(P<0.001)$ (Table 2$)$.

\section{Discussion}

In the present study, the results showed that the combination of $\mathrm{PNI}^{\mathrm{P} 3-\mathrm{P} 1}$ ratio and serum amylase level on POD 1 was a useful indicator for prediction of POPF following pancreaticoduodenectomy. Additionally, our results support the notion that both systemic response-related indicators and local responserelated indicators reflect the development of POPF following pancreaticoduodenectomy. POPF following pancreaticoduodenectomy is a serious complication, which leads to poor long-term surgical outcomes, prolonged hospital stay, and increased medical costs. POPF also carries a poor prognosis in malignant disease, secondary to systemic immune function exhaustion or delayed adjuvant therapy [20-22]. Extensive

Table 2 Univariate and multivariate analyses of the predictive indicators of POPF in patients who underwent pancreaticoduodenectomy

\begin{tabular}{|c|c|c|c|c|c|c|}
\hline \multirow[t]{2}{*}{ Variables } & \multicolumn{3}{|c|}{ Univariate analysis } & \multicolumn{3}{|c|}{ Multivariate analysis } \\
\hline & Odds ratio & $95 \% \mathrm{Cl}$ & $P$ value & Odds ratio & $95 \% \mathrm{Cl}$ & $P$ value \\
\hline Age ( $\geq 70$ vs. $<70)$ & 0.704 & $0.374-1.324$ & 0.276 & & & \\
\hline Sex (male vs. female) & 2.614 & $1266-5.396$ & 0.009 & 2.514 & $0.916-6.902$ & 0.074 \\
\hline Body mass index ( $\geq 25$ vs. $<25$ ) & 2.738 & $1.252-5.987$ & 0.012 & 0.896 & $0.265-3.025$ & 0.896 \\
\hline Preoperative biliary drainage (present vs. absent) & 2.005 & $1.060-3.791$ & 0.032 & 3.313 & $1.216-9.053$ & 0.019 \\
\hline Pancreatic texture of remnant pancreas (soft vs. hard) & 10.088 & $3.791-26.848$ & $<0.001$ & 2.607 & $0.533-12.745$ & 0.237 \\
\hline Diameter of main pancreatic duct ( $\geq 3 \mathrm{~mm}$ vs. $<3 \mathrm{~mm}$ ) & 4.070 & 2.094-7.911 & $<0.001$ & 1.396 & $0.535-3.643$ & 0.495 \\
\hline Operative time ( $\geq 480 \mathrm{~min}$ vs. $<480 \mathrm{~min}$ ) & 1.476 & $0.719-3.031$ & 0.289 & & & \\
\hline Intraoperative blood loss ( $\geq 1000 \mathrm{ml}$ vs. $<1000 \mathrm{ml}$ ) & 1.663 & $0.784-3.523$ & 0.185 & & & \\
\hline Pancreatic stent (internal stent vs. external stent) & 0.401 & $0.182-0.887$ & 0.024 & 0.378 & $0.121-1.178$ & 0.094 \\
\hline Drain amylase level on POD 1 ( $\geq 4000$ IU/I vs. < 4000 IU/I) & 7.727 & $3.745-15.941$ & $<0.001$ & 2.565 & $0.632-10.417$ & 0.188 \\
\hline Serum CRP level on POD 3 ( $\geq 17.7$ mg/dl vs. $<17.7$ mg/dl) & 12.226 & $5.782-25.850$ & $<0.001$ & 6.929 & $2.458-19.534$ & $<0.001$ \\
\hline Combination of PNI ${ }^{\mathrm{P3}-\mathrm{P} 1}$ ratio and serum amylase level on POD 1 & & & $<0.001$ & & & 0.038 \\
\hline score 0 vs. score 2 & 44.571 & $5.732-346.559$ & $<0.001$ & 17.661 & $1.751-178.140$ & 0.015 \\
\hline score 1 vs. score 2 & 17.778 & $2.299-137.497$ & 0.006 & 9.411 & $0.961-92.211$ & 0.054 \\
\hline
\end{tabular}

$\mathrm{Cl}$ confidence interval, $B A$ Blumgart anastomosis, $P O D$ postoperative day, $P N I$ prognostic nutritional index 
efforts have been made to improve surgical outcomes following pancreaticoduodenectomy; however, there are no definitive methods to prevent POPF. Therefore, although POPF cannot be completely avoided, accurate prediction of POPF early after pancreaticoduodenectomy is needed to prevent secondary lifethreatening complications such as intra-abdominal abscess or postpancreatectomy hemorrhage.

PNI as a predictive indicator of POPF was the focus of this study because perioperative nutritional status is an important factor closely associated with postoperative surgical outcomes [23]. PNI is calculated using serum albumin level and peripheral total lymphocyte count; it was originally used to assess perioperative nutritional conditions and postoperative complications in patients with cancer [4]. Albumin is a negative acute-phase marker synthesized by the liver; its levels decrease in the presence of inflammation [24]. Inflammation increases capillary permeability and leakage of serum albumin, leading to expansion of the interstitial space and increasing the distribution volume of albumin. Additionally, the half-life of albumin has been shown to decrease, thereby reducing total albumin mass [25]. Hypoalbuminemia is associated with poor tissue healing, decreased collagen synthesis at anastomoses, and impaired cell-mediated immunity (e.g., macrophage activation and granuloma formation) [26, 27]. Additionally, peripheral total lymphocyte count reflects inflammation and immunity, as well as nutritional status [28-30]; therefore, PNI reflects patients' systemic response secondary to inflammation, nutritional status, and immunity. Low perioperative PNI reportedly affects the complication rate after pancreaticoduodenectomy, including POPF [7, 8, 31]. Previous studies have indicated the usefulness of PNI perioperatively for prediction of complications after pancreaticoduodenectomy, including POPF. However, the hemodynamics of the inflammatory response occur dynamically; dynamic changes in PNI during the early postoperative period might be more desirable than PNI postoperatively for prediction of POPF following pancreaticoduodenectomy. In fact, previous reports indicated that a steep rise in serum CRP levels from POD 1 to POD 3 was a highly predictive factor for subsequent POPF following pancreaticoduodenectomy [32]. Our results showed that the $\mathrm{PNI}^{\mathrm{P} 3-\mathrm{P} 1}$ ratio was a useful indicator for prediction of POPF following pancreaticoduodenectomy, reflecting the comprehensive hemodynamic response related to nutrition, inflammation, and immune activity in patients undergoing pancreaticoduodenectomy.

Compared with PNI, serum CRP and albumin are more widely accepted systemic inflammatory markers; in this study, CRP on POD3 was confirmed as an independent predictor of POPF. However, albumin is inferior to PNI for prediction of POPF after pancreaticoduodenectomy because PNI consists of both albumin and lymphocytes. Regarding serum CRP, there are individual differences in the distributions of serum CRP levels related to genetic or environmental factors; there is also a significant association between regulation of serum CRP levels and the presence of polymorphisms in the promoter of interleukin-6 [33]. Therefore, PNI might be more suitable than serum CRP or albumin for prediction of POPF as a factor that changes dynamically during the early postoperative period.

In general, drain amylase level is well-recognized as a predictive factor for POPF; high drain amylase level $(\geq$ $4000 \mathrm{IU} / \mathrm{l}$ ) on POD1 is reportedly an independent predictive risk factor for the development of POPF [19]. In contrast, several studies indicated the usefulness of postoperative serum amylase level for prediction of POPF following pancreaticoduodenectomy [13, 14, 34-37]. Our results also showed that an elevated serum amylase level on POD 1 was significantly associated with POPF. The mechanism underlying hyperamylasemia in patients with POPF during the early postoperative period after pancreaticoduodenectomy is explained by the finding that postoperative acute pancreatitis, based on elevated serum amylase level on either POD 0 or 1 , might be a biochemical manifestation of intraoperative ischemic damage to the pancreatic stump that eventually leads to POPF $[38,39]$. For this reason, serum amylase level during the early postoperative period may reflect the local inflammatory response to pancreaticojejunostomy after pancreaticoduodenectomy.

PNI ratio and serum amylase level during the early postoperative period indicate different patient responses after pancreaticoduodenectomy; these two indicators can help to predict POPF following pancreaticoduodenectomy. Almost no correlation between $\mathrm{PNI}^{\mathrm{P} 3-\mathrm{P} 1}$ ratio and serum amylase level on POD 1 was observed in this study. These results led us to consider that, for prediction of POPF after pancreaticoduodenectomy, the combination of these two indicators would be superior to either measure alone. In fact, the AUC for the combination of $\mathrm{PNI}^{\mathrm{P} 3-\mathrm{P} 1}$ ratio and serum amylase level on POD 1 was higher than that for either value separately, as well as for drain amylase level on POD1; our results revealed that the combination of $\mathrm{PNI}^{\mathrm{P} 3-\mathrm{P} 1}$ ratio and serum amylase level on POD 1 was an independent predictive indicator for POPF following pancreaticoduodenectomy.

There were some limitations in our study. First, this was a retrospective analysis, which might have involved bias. Second, we measured PNI before surgery, as well as on POD 1 and 3, and measured serum amylase levels on POD 1 and 3; the optimal times for measurement of these indicators are unclear. Third, PNI after surgery might have been affected by intraoperative 
administration of exogenous intravenous albumin or blood transfusion in some patients. Fourth, the number of patients included in this study was small; a largescale, prospective study is needed to verify our results.

\section{Conclusions}

In conclusion, the range of change in PNI from POD 1 to POD 3 was significantly associated with POPF following pancreaticoduodenectomy. Furthermore, the combination of the range of change in PNI from POD 1 to POD 3 and serum amylase levels on POD 1 were useful indicators for prediction of POPF following pancreaticoduodenectomy. These two indicators, inexpensively measured from peripheral blood samples during the early postoperative period, could quickly predict POPF following pancreaticoduodenectomy and might prevent lifethreatening complications.

\section{Abbreviations}

POPF: Postoperative pancreatic fistula; PNI: Prognostic nutritional index; POD: Postoperative day; CRP: C-reactive protein; AUC: Area under the curve

\section{Acknowledgements}

We thank Jane Charbonneau, DVM, and Ryan Chastain-Gross, PhD, from Edanz Group (https://en-author-services.edanzgroup.com) for editing a draft of this manuscript.

\section{Authors' contributions}

Study conception: TS; Study design: TS; Data acquisition: TY, EU, MM, and TH; Quality control of data and algorithms: JW; Data analysis and interpretation: TS and NT; Statistical analysis: TS; Manuscript preparation: TS; Manuscript editing: SH; Manuscript review: YF; Final approval of the article: all authors.

\section{Funding}

There was no funding source.

\section{Availability of data and materials}

The datasets used and analyzed during the current study are available from the corresponding author on reasonable request.

\section{Ethics approval and consent to participate}

All procedures performed in this study involving human participants were in accordance with the ethical standards of the institutional review board of ethics committee and national research committee with the 1964 Helsinki declaration and its later amendments. The Tottori University Hospital Ethics Committee approved this study (No. 17A135).

\section{Consent for publication}

Not applicable.

\section{Competing interests}

The authors declare that they have no competing interests.

Received: 24 December 2019 Accepted: 30 July 2020

Published online: 06 August 2020

\section{References}

1. Aoki S, Miyata H, Konno H, Gotoh M, Motoi F, Kumamaru H, et al. Risk factors of serious postoperative complications after pancreaticoduodenectomy and risk calculators for predicting postoperative complications: a nationwide study of 17,564 patients in Japan. J Hepatobiliary Pancreat Sci. 2017;24:243-51.

2. Zhang GQ, Li XH, Ye XJ, Chen HB, Fu NT, Wu AT, Li Y. Internal versus external drainage with a pancreatic duct stent for Pancreaticojejunostomy during Pancreaticoduodenectomy for patients at high risk for pancreatic fistula: a comparative study. J Surg Res. 2018;232:247-56.
3. Fu SJ, Shen SL, Li SQ, Hu WJ, Hua YP, Kuang M, et al. Risk factors and outcomes of postoperative pancreatic fistula after pancreaticoduodenectomy: an audit of 532 consecutive cases. BMC Surg. 2015;15:34 Published 2015 Mar 26.

4. Onodera T, Goseki N, Kosaki G. Prognostic nutritional index in gastrointestinal surgery of malnourished cancer patients. Nihon Geka Gakkai Zasshi. 1984;85(9):1001-5.

5. Oshi M, Kunisaki C, Miyamoto H, Kosaka T, Akiyama H, Endo I. Risk factors for anastomotic leakage of Esophagojejunostomy after laparoscopy-assisted Total Gastrectomy for gastric Cancer. Dig Surg. 2018;35(1):28-34.

6. Wang $X$, Wang $Y$. The prognostic nutritional index is prognostic factor of gynecological cancer: a systematic review and meta-analysis. Int J Surg. 2019;67:79-86.

7. Sato N, Tamura T, Minagawa N, Hirata K. Preoperative body mass index-toprognostic nutritional index ratio predicts pancreatic fistula after pancreaticoduodenectomy. Hepatobiliary Surg Nutr. 2016;5:256-62.

8. Rungsakulkij N, Tangtawee P, Suragul W, Muangkaew P, Mingphruedhi S, Aeesoa S. Correlation of serum albumin and prognostic nutritional index with outcomes following pancreaticoduodenectomy. World J Clin Cases. 2019;7:28-38.

9. Bassi C, Dervenis C, Butturini G, Fingerhut A, Yeo C, Izbicki J, et al. Postoperative pancreatic fistula: an international study group (ISGPF) definition. Surgery. 2005;138:8-13.

10. Shinchi H, Wada K, Traverso LW. The usefulness of drain data to identify a clinically relevant pancreatic anastomotic leak after pancreaticoduodenectomy? J Gastrointest Surg. 2006;10:490-8.

11. Gebauer F, Kloth K, Tachezy M, Vashist YK, Cataldegirmen G, Izbicki JR, et al. Options and limitations in applying the fistula classification by the international study Group for Pancreatic Fistula. Ann Surg. 2012;256:130-8.

12. Chen CB, McCall NS, Pucci MJ, Leiby B, Dabbish N, Winter JM, et al. The combination of pancreas texture and postoperative serum amylase in predicting pancreatic fistula risk. Am Surg. 2018;84(6):889-96.

13. Cloyd JM, Kastenberg ZJ, Visser BC, Poultsides GA, Norton JA. Postoperative serum amylase predicts pancreatic fistula formation following pancreaticoduodenectomy. J Gastrointest Surg. 2014;18:348-53. https://doi. org/10.1007/s11605-013-2293-3.

14. Palani Velu LK, Chandrabalan W, Jabbar S, McMillan DC, McKay CJ, Carter $C R$, et al. Serum amylase on the night of surgery predicts clinically significant pancreatic fistula after pancreaticoduodenectomy. HPB (Oxford). 2014;16:610-9.

15. Raty S, Sand J, Lantto E, Nordback I. Postoperative acute pancreatitis as a major determinant of postoperative delayed gastric emptying after pancreaticoduodenectomy. J Gastrointest Surg. 2006;10:1131-9.

16. Ansorge C, Regner S, Segersvard R, Strommer L. Early intraperitoneal metabolic changes and protease activation as indicators of pancreatic fistula after pancreaticoduodenectomy. Br J Surg. 2012;99:104-11.

17. Fujii T, Sugimoto H, Yamada S, Kanda M, Suenaga M, Takami H, et al. Modified Blumgart anastomosis for pancreaticojejunostomy: technical improvement in matched historical control study. J Gastrointest Surg. 2014; 18:1108-15

18. Bassi C, Marchegiani G, Dervenis C, Sarr M, Abu Hilal M, Adham M, et al. The 2016 update of the international study group (ISGPS) definition and grading of postoperative pancreatic fistula: 11 years after. Surgery. 2017;161:584-91.

19. Kawai M, Kondo S, Yamaue H, Wada K, Sano K, Motoi F, et al. Predictive risk factors for clinically relevant pancreatic fistula analyzed in 1,239 patients with pancreaticoduodenectomy: multicenter data collection as a project study of pancreatic surgery by the Japanese Society of Hepato-BiliaryPancreatic Surgery. J Hepatobiliary Pancreat Sci. 2011;18:601-8.

20. Smits FJ, van Santvoort HC, Besselink MG, Batenburg MCT, Slooff RAE, Boerma D, et al. Management of Severe Pancreatic Fistula after Pancreatoduodenectomy. JAMA Surg. 2017;152:540-8.

21. Brown EG, Yang A, Canter RJ, Bold RJ. Outcomes of pancreaticoduodenectomy: where should we focus our efforts on improving outcomes? JAMA Surg. 2014;149:694-9.

22. Watanabe $Y$, Nishihara $K$, Matsumoto $S$, Okayama $T$, Abe $Y$, Nakano T. Effect of postoperative major complications on prognosis after pancreatectomy for pancreatic cancer: a retrospective review. Surg Today. 2017:47:555-67.

23. Costa MD, Vieira de Melo CY, Amorim AC, Cipriano Torres Dde O, dos Santos AC. Association between nutritional status, inflammatory condition, and prognostic indexes with postoperative complications and clinical 
outcome of patients with gastrointestinal Neoplasia. Nutr Cancer. 2016;68: 1108-14.

24. Matowicka-Karna J. Markers of inflammation, activation of blood platelets and coagulation disorders in inflammatory bowel diseases. Postepy Hig Med Dosw. 2016;70:305-12.

25. Soeters PB, Wolfe RR, Shenkin A. Hypoalbuminemia: pathogenesis and clinical significance. JPEN J Parenter Enteral Nutr. 2019;43:181-93.

26. Rivadeneira DE, Grobmyer SR, Naama HA, Mackrell PJ, Mestre JR, Stapleton PP, et al. Malnutrition-induced macrophage apoptosis. Surgery. 2001;129: $617-25$.

27. Reynolds JV, Redmond HP, Ueno N, Steigman C, Ziegler MM, Daly JM, et al. Impairment of macrophage activation and granuloma formation by protein deprivation in mice. Cell Immunol. 1992;139:493-504.

28. Kim EY, Hong TH. Changes in total lymphocyte count and neutrophil-tolymphocyte ratio after curative pancreatectomy in patients with pancreas adenocarcinoma and their prognostic role. J Surg Oncol. 2019;120(7):1102-11.

29. Iseki Y, Shibutani M, Maeda K, Nagahara H, Tamura T, Ohira G, et al. The impact of the preoperative peripheral lymphocyte count and lymphocyte percentage in patients with colorectal cancer. Surg Today. 2017;47(6):743-54.

30. Chen JH, Zhai ET, Yuan YJ, Wu KM, Xu JB, Peng JJ, et al. Systemic immuneinflammation index for predicting prognosis of colorectal cancer. World J Gastroenterol. 2017;23(34):6261-72.

31. Kanda M, Fujii T, Kodera Y, Nagai S, Takeda S, Nakao A. Nutritional predictors of postoperative outcome in pancreatic cancer. Br J Surg. 2011;98:268-74.

32. Kanda M, Fujii T, Takami H, Suenaga M, Inokawa Y, Yamada S, et al. Novel diagnostics for aggravating pancreatic fistulas at the acute phase after pancreatectomy. World J Gastroenterol. 2014;20:8535-44.

33. Okada Y, Takahashi A, Ohmiya H, Kumasaka N, Kamatani Y, Hosono N, et al. Genome-wide association study for C-reactive protein levels identified pleiotropic associations in the IL6 locus. Hum Mol Genet. 2011;20(6):1224-31.

34. Winter JM, Cameron JL, Yeo CJ, Alao B, Lillemoe KD, Campbell KA, et al. Biochemical markers predict morbidity and mortality after pancreaticoduodenectomy. J Am Coll Surg. 2007;204:1029-36 discussion 1037-1028.

35. Jin S, Shi XJ, Wang SY, Zhang P, Lv GY, Du XH, et al. Drainage fluid and serum amylase levels accurately predict development of postoperative pancreatic fistula. World I Gastroenterol. 2017;23:6357-64.

36. Okabayashi T, Kobayashi M, Nishimori I, Sugimoto T, Onishi S, Hanazaki K. Risk factors, predictors and prevention of pancreatic fistula formation after pancreatoduodenectomy. J Hepato Biliary Pancreat Surg. 2007;14:557-63.

37. Smits FJ, Molenaar IQ, Besselink MG, Borel Rinkes IHM, van Eijck CHJ, Busch $\mathrm{OR}$, et al. Early recognition of clinically relevant postoperative pancreatic fistula: a systematic review. HPB (Oxford). 2019. https://doi.org/10.1016/j.hpb. 2019.07.005

38. Connor S. Defining post-operative pancreatitis as a new pancreatic specific complication following pancreatic resection. HPB (Oxford). 2016;18:642-51.

39. Bannone E, Andrianello S, Marchegiani G, Masini G, Malleo G, Bassi C, et al. Postoperative acute pancreatitis following Pancreaticoduodenectomy: a determinant of fistula potentially driven by the intraoperative fluid management. Ann Surg. 2018;268:815-22.

\section{Publisher's Note}

Springer Nature remains neutral with regard to jurisdictional claims in published maps and institutional affiliations.

Ready to submit your research? Choose BMC and benefit from:
- fast, convenient online submission
- thorough peer review by experienced researchers in your field
- rapid publication on acceptance
- support for research data, including large and complex data types
- gold Open Access which fosters wider collaboration and increased citations
- maximum visibility for your research: over 100M website views per year
At BMC, research is always in progress.
Learn more biomedcentral.com/submissions

